\title{
Discrete Structures in the CS Envelope of the Be star $\zeta$ Tau
}

D. Ballereau ${ }^{1}$, J. Chauville ${ }^{1}$, H. Cao ${ }^{2}$, M. Floquet ${ }^{1}$, A.M. Hubert ${ }^{1}$ and J. Zorec ${ }^{3}$

${ }^{1}$ Dasgal, UMR8633 du CNRS, Observatoire de Paris-Meudon, F-92195 Meudon, France

\section{${ }^{2}$ Beijing Astronomical Observatory, Chinese Academy of Sciences, China}

${ }^{3}$ Institut d'Astrophysique de Paris - CNRS, 98bis Bd Arago, 75014-Paris, France

\begin{abstract}
The recent cycle of the $\mathrm{V} / \mathrm{R}$ ratio and $\mathrm{RV}$ variations in $\zeta$ Tau has been found similar to that reported by Delplace (1970). As a rule, in both cycles, singly-ionized metallic shell lines display discrete components when the descending branch of RV curve of Balmer lines crosses the stellar RV value; a narrow sharp feature is mainly superimposed on a broad and bluer one, both being slowly varying over some months; very weak additional components are suspected on stronger Fe II lines. On another hand, in the ascending branch of RV curve (RV $\geq R V_{\text {star }}$ ), secondary shell components varying in intensity are conspicuous on $\mathrm{V}$ and $\mathrm{R}$ emissions of the first Balmer lines.
\end{abstract}

\section{Introduction}

The Be shell star $\zeta$ Tau is well known to show V/R ratio cycles associated with shell line radial velocity and profile variations. Common features with two other shell stars $48 \mathrm{Lib}$ and EW Lac are summarized in Hubert et al. (1987 and ref. therein).

Long-term variations in $\zeta$ Tau were many times reported in the literature. The amplitude and the duration of each cycle (V/R ratio, RV) was found to be variable since 1954. Mon et al. (1992), then Guo et al. (1995) observed a minimum of variability between 1983 and 1988, with a random fluctuation of the $\mathrm{V} / \mathrm{R}$ ratio. According to these authors a new series of cycles started in 1988 as the equivalent width of $\mathrm{H} \alpha$ emission increased from $6 \AA$ to $24 \AA$ in 1992, then fluctuated around this value.

As for $48 \mathrm{Lib}$ and EW Lac a composite structure in shell lines of $\zeta$ Tau (doubling of metallic lines; blue and red shell satellites in low Balmer lines) was observed at some located phases in the V/R ratio and RV cycles. The complex structure of Fe II red lines in Be stars has been emphasized with high S/N spectra (Hanuschik et al. 1996). 


\section{Observations}

Observations reported in this study were made in different wavelength ranges from 1992 to 1998 :

OHP : $1.52 \mathrm{~m}$ telescope - spectrograph AURELIE $(11000<\mathrm{R}<24000)-29 \mathrm{sp}$. $1.93 \mathrm{~m}$ telescope - spectrometer ELODIE $(\mathrm{R}=43000)-1 \mathrm{sp}$.

Xinglong: $2.16 \mathrm{~m}$ telescope - echelle spectrograph $(\mathrm{R}=35000$ at $\mathrm{H} \alpha ; \mathrm{R}=26000$ at $\mathrm{H} \beta) ; 37 \mathrm{sp}$.

ESO:1.52m telescope - echelle spectrograph $(R=35000)$ - 1 sp.

\section{Results}

The duration of the last V/R and RV cycles is about 6 years, quite comparable to that observed in the sixties (Delplace 1970) and as a rule, the behaviour of shell and emission line profiles is similar. The RV semi-amplitude is about 80 $\mathrm{km} / \mathrm{s}$. (The orbital contribution in RV measurements was taken off using the solution for the HI lines given by Harmanec, 1984).

\subsection{Metallic shell line doubling}

A metallic line doubling is observed (Figure $1 \mathrm{a}, \mathrm{b}$ ) when $R V_{\text {shell }}$ crosses $R V_{\text {star }}$ in the descending branch of the curve and in the $\mathrm{V} / \mathrm{R}<1$ phase; the superposition of a broad blue-shifted and a narrow component is conspicuous on ?some? spectra. Both are variable in intensity and radial velocity. In the ascending branch of the positive RV curve, metallic lines are strongly red-winged.

\subsection{Blue and red $\mathbf{H}$ satellite shell lines}

High resolution spectra nicely show the presence of satellite shell components on $\mathrm{H} \alpha$ and $\mathrm{H} \beta$ in the ascending branch of $R V_{\text {shell }}$ curve (Figure 2), when $R V_{\text {shell }}$ crosses $R V_{\text {star }}$, and after in the first part of the $V / R>1$ phase. On the spectrum taken on $1994 / 08 / 28$, a narrow red satellite shell line $(+95 \mathrm{~km} / \mathrm{s})$ is seen on the first four Balmer lines. A strong difference in the shape and in the $V / R$ ratio of $\mathrm{H} \alpha$ and $\mathrm{H} \beta$ can be noted. In 1994 August and November no main central shell component is seen on $\mathrm{H} \alpha$, the line appears single like with a red satellite shell component; this last is shifted to $+137 \mathrm{~km} / \mathrm{s}$ in November. In 1995 February blue and red satellites with respectively radial velocities $-140 \mathrm{~km} / \mathrm{s}$ and +130 $\mathrm{km} / \mathrm{s}$, are seen on $\mathrm{H} \alpha$ and $\mathrm{H} \beta$. In 1995 November and December the blue component $(-75 \mathrm{~km} / \mathrm{s})$ is stronger on both lines as the red one $(+174 \mathrm{~km} / \mathrm{s})$ is weaker and only observed on $\mathrm{H} \beta$, then this latter component has disappeared in the spectrum taken in 1996 January. The blue satellite in turn has disappeared from both lines by the end of February 1996. On $\mathrm{H} \beta$ the red satellite was observed for one and a half years at least, and the blue one for a year at least. Both moved towards long wavelengths.

\subsection{Unidentified emission lines?}

Broad and double emission lines are seen at 6318 and $6384 \AA$. These features are also present in $48 \mathrm{Lib}$ and in other Be stars with strong Fe II emission lines and 

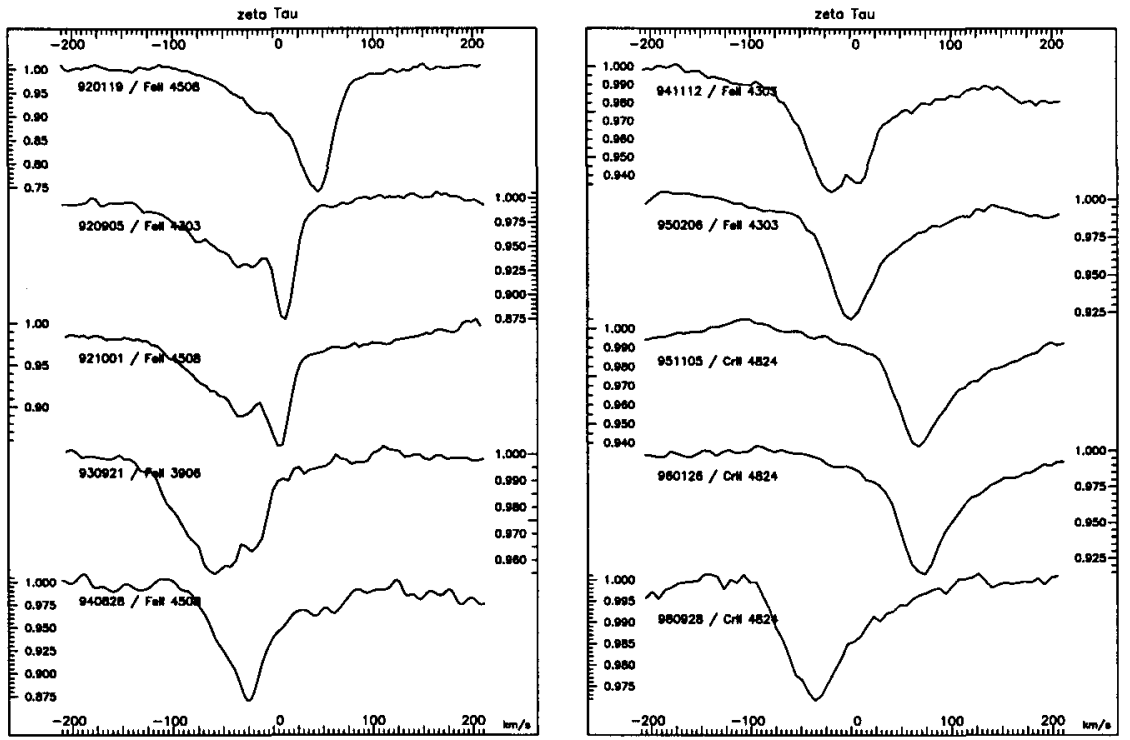

Figure 1. Composite structure of metallic shell lines across RV and $\mathrm{V} / \mathrm{R}$ ratio cycles

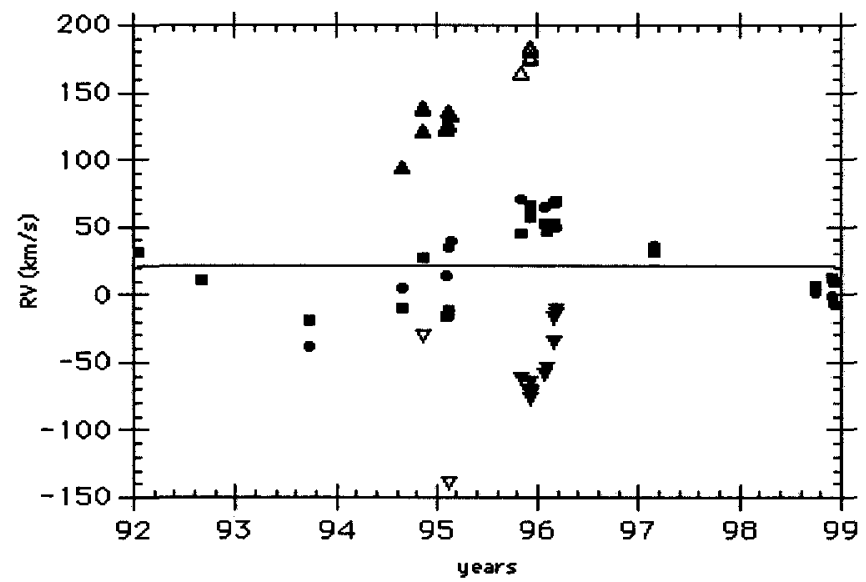

Figure 2. RV cycle for shell lines. $\mathrm{H} 3, \mathrm{H} 4, \mathrm{H} 5$; main component: filled squares; blue and red satellites (open if weak, filled if strong): triangle down and triangle up respectively. He I 5876, $6678 \AA$ : filled circles. Straight line: $R V_{\text {star }}=+22 \mathrm{~km} / \mathrm{s}$; years $=1900+$ 
in $\mathrm{B}[\mathrm{e}]$ stars such as $\mathrm{HD}$ 50138. These lines are probably due to unclassified Fe II lines. In Be stars with a moderate $v \sin i$, lines at 6318 and $6384 \AA$ have generally the same structure as the Fe II lines of multiplets 48 and 49 . In $\zeta$ Tau this is true for the $\mathrm{V}$ and $\mathrm{R}$ emission components, but differently the central shell commonly observed in metallic lines (such as Fe II, Si II) is not present at 6318 and $6384 \AA$. It is then suggested that these later lines are formed in low opacity regions which are probably not very extended, and/or outside the part of the disc projected on the star. An interferometric investigation in these lines could help to understand their formation region and also those of metallic lines in general.

\section{Discussion}

Blue and red shell satellites seen on $\mathrm{H} \alpha$ and $\mathrm{H} \beta$ from mid-1994 to early 1996 seem to result from an occultation phenomenon produced by different inhomogeneous parts of the disc which progressively hid each other; the red satellite disappears prior to the blue one indicating a rotation of inhomogeneities in the same sense as the disc. In the frame of an one-armed disc model, satellites occur when the density wave moves, in the line of sight, behind the star (ascending branch of the RV curve when $R V_{\text {shell }}$ crosses $R V_{\text {star }}$ ). We note however that the blue shell satellite on low Balmer terms, metallic lines and the main shell component on $\mathrm{H} \beta, \mathrm{H} \gamma, \mathrm{H} \delta$ are strongly red-shifted (RV $\sim 70 \mathrm{~km} / \mathrm{s}$ ) and close to a maximum in the RV curve. Weak metallic lines are purely in absorption, their shift towards the long wavelengths do not result from an apparent composition of a strong red and a weak blue emission peaks. Therefore these lines cannot be formed in the same regions disturbed by the density wave where $\mathrm{H}$ shell satellites could originate.

The structure of hydrogen lines: inverse P Cyg like profiles, where the emission component has two peaks is reminiscent of the presence of a moving ring. The presence of a compound velocity field evokes hence a complex problem of interacting winds with the circumstellar medium.

\section{References}

Delplace, A.M. 1970, A\&A 7, 459

Guo, Y., Huang L., Hao, J. et al. 1995, A\&AS 112, 201

Hanuschik, R.W., Hummel, W., Sutorius, E. et al. 1996, A\&AS 116, 309

Harmanec, P. 1984, Bull. Astron. Inst. Czech. 35, 164

Hubert, A.M., Floquet, M., Chambon, M.T. 1987, A\&A 186, 213

Mon, M., Kogure, T., Suzuki, M. et al. 1992, PASJ 44, 73 